\title{
Digital Humanities: to a question of the directions and prospects of development of interdisciplinarity in humanitarian researches and education
}

\author{
Galina Mozhaeva ${ }^{1 a}$, Polina Mozhaeva Renha ${ }^{1}$ \\ ${ }^{1}$ Tomsk State University, Tomsk, 634050, Russian Federation
}

\begin{abstract}
In this work the results of the research on Digital Humanities (DH) as interdisciplinary field of researches are presented, the main digitalization directions of the humanities in the modern world are defined. The empirical material was built and verified, on which base 323 scientific and educational structures positioning themselves in the field of $\mathrm{DH}$ in Europe, Asia, North and South America, Australia and Oceania are revealed. Organizational forms and infrastructure of researches in the field of DH in the leading world centers are studied. The analysis of the key directions in researches and development in the field of DH is carried out. The quantitative analysis of basic data was made. As a result of analysis 46 main activities of DH centers are revealed, which are localized on the world map: http://huminf.tsu.ru/nir/dh/map.htm.
\end{abstract}

Keywords: Digital humanities; modern technology of humanitarian studies; research infrastructure; world digital humanities centers.

\section{Introduction}

Digital humanities (further - DH) is the interdisciplinary field of researches that combines methods and practices of humanitarian, social and computational sciences in order to explore the possibilities of the application and interpretation of new digital and informationcommunicational technologies in the humanities and education. During an era of the digital technologies DH became one of the innovative directions of development of the humanities which promotes to consolidation and self-organization of researchers in various sciences, to development of the general principles, methods, and scientific digital tools.

\footnotetext{
${ }^{\text {a }}$ Corresponding author: mozhaeva@ido.tsu.ru
} 
This perspective scientific direction is widely adopted at the majority of leading universities of the world and only begins the development in Russia. There are a lot more researchers who intentionally ignore IT potential for the humanities and consider that IT destroys modern humanitarian knowledge. At the same time, successful researches in the field of branch informatics, based on application IT as tool for researches of traditional material are conducted at many Russian universities and scientific institutes.

It is necessary to define the main directions of digitalization of the humanities in the modern world, to reveal the general and special in comparison of the Russian humanitarian centers with the foreign $\mathrm{DH}$ centers and to define the most perspective and actual $\mathrm{DH}$ directions in Russia for understanding of prospects of development of DH in Russia.

\section{Short review of researches}

The number of the researches, devoted to various aspects of development of $\mathrm{DH}$, grows. Consolidation and self-organization of researchers within DH is observed, "There is an elaboration of the general principles, methods, and scientific digital tools", [7].

Professor of the Cologne University (Germany) Manfred Taller made the analysis of a current state of the interdisciplinary DH area in his work "Discussions around Digital Humanities". M. Taller noted that "conceptual decisions about using of new technologies in the humanities must be defined by concepts of the humanities", [4].

According to specialists of the Californian University UCLA, Digital Humanities interprets the cultural and social impact of new media and information technologies, as well as creates and applies these technologies to interrogate cultural, social, historical, and philological questions, [6].

In Russia steady interest of researchers to the new direction was designated within Russian branch of the International Association «History and Computing». The participants of this Association investigate problems and prospects of DH in the context of development of methodology of historical informatics, [3]. The project «Humanities in the digital age: from branch informatics to Digital Humanities» is directed on research of current trends of DH development, [1].

\section{Methodology}

The logic of development of the humanities moves today in the direction of methodological interdisciplinarity, search of general scientific methodology which would allow not simply to unite tools of separate sciences, but to develop the general bases for humanitarian and natural-science researches.

As general scientific methodology of DH we consider the theory of information which was developed in the 1940-50th years for technical sciences, and in the 1970th years took shape as general scientific methodology. The theory of information allows not only to go beyond technocratic approach to understanding of essence of information, but also to prove need of interdisciplinary integration researches. This theory allows to see that synthesis of approaches to studying of information is possible only in the humanitarian sphere on an interdisciplinary basis that is shown today in development of $\mathrm{DH}$ which transforms humanitarian researches via digital tools and resources. Combination of various technologies and methods, expert knowledge becomes a key for DH where are broken "disciplinary" borders, and subjects, meanings, searches, decisions intertwine, [5]. 


\section{Methods}

The analysis of the main of aspects of development of DH is carried out for the solution of this task. Modern sociological methods, statistical data processing, etc. are applied in this work. The automated environments were used for data collection. The analysis of integrity and completeness of information, the analysis of spatial and temporary compliances were used for the comparative analysis. The applied methods of the DH (statistical data processing, the content analysis, elements of GIS technologies, visualization) are applied for representation of results of research.

Information search about the Digital Humanities centers was carried out in the Internet where are placed hundreds of specialized web pages, accounts in the social networks and social media devoted to Digital Humanities, a number of special articles, books, periodicals [2], the series of publications united in research projects, etc.,[7].

For the purpose of establishment of reliability of collected empirical materials the verification of information sources revealed as a result of search queries in the Internet was carried out.

\section{Results}

On the basis of the preliminary analysis of literature some search queries for information search on the Internet were formulated: Digital Humanities, Humanities Computing, Humanités digitales, Humanités numériques, Humanistisk Informatik, Humanidades Digitales, Computing in the Humanities, Digital Culture, Internet Studies, Information Studies, eHistory, eHumanities, eScience, humanitarian informatics, Social Robotics, etc.

More than 1500 results of search queries on the Internet in English, French, Portuguese, Spanish, German and Russian languages and data from the research works published in the press were made. During research empirical material was built and verified.

323 scientific and educational structures positioning themselves in the field of DH in Europe, Asia, North and South America, Australia and Oceania are revealed.

\subsection{Infrastructure of researches in the field of DH}

Organizational forms and infrastructure of researches in the field of DH in the leading world centers are studied.

The analysis of collected information showed that the most widespread organizational form is the center (center, centre) (114 structures that makes $35 \%$ of total). 49 structures carry the name of laboratories (lab, laboratory) (15\%). 43 and 17 structures respectively carry themselves to institutes and departments (chairs, faculties) that makes $14 \%$ and $5 \%$ respectively. "Groups" and "schools" account for 4\% of the total (13 and 12 structures respectively). 3\% (10 structures) call themselves "initiatives". All structures with other names numbering less than 10 representatives were carried by us in the category "other organizational forms" which made $20 \%$. These include such structures as library, project, association, network, fund, archive, studio, platform, collaboration, section, society, consortium, etc.

Results of the analysis and processing of empirical data showed that the structures allocated for the analysis positioning the belonging to the DH area are geographically distributed as follows:

- in Europe - 152 structures with a dominant in Great Britain (23), France (27) and Germany (20); 
- in Asia - 16 structures with a dominant in Japan (5), China (4) and Singapore (3);

- in North America - 134 structures, including 115 in the USA and 18 in Canada;

- in South America - 9 structures, including 7 in Brazil and 2 in Argentina;

- in Australia and Oceania - 12 structures, including 9 in Australia and 3 in New Zealand.

Visually the interactive map "The world centers Digital Humanities" which prototype is created within this research allows to present geography of distribution of the studied structures in the field of Digital humanities. Visualization of infrastructure of the digital humanities in the world allowed not only to find geographical points of concentration of the revealed 323 structures positioning themselves in the field of Digital Humanities but also to accompany points of their placement on the card with the reference information including the name, the short description of activity, date of creation, the address, a name and contacts of the head, the link to the website of the center.

It is possible to study the working version of a prototype of the interactive map "The world centers Digital Humanities" created by means of an open cartographical online service of Google Maps Engine and allowing to visualize infrastructure of the digital humanities in the world to the address: http://huminf.tsu.ru/nir/dh/map.htm.

\subsection{The main directions of scientific and educational activity in DH}

The analysis of the key directions in researches and development in the field of DH is carried out.

The majority of the considered structures supports interdisciplinary researches in the educational institution, advances application of digital technologies for humanitarian researches, advises and gives technical assistance to humanists, regularly holds seminars and master classes in subjects of DH.

As a result of the analysis of 323 scientific and educational structures working in the field of DH 46 main activities which group allowed to define are revealed that 26 from them have the widest circulation in structures of $\mathrm{DH}$.

The most widespread activity is implementation of educational programs and courses in the sphere of Digital Humanities (master and postgraduate programs, short-term trainings and courses) (85 structures).

Among other DH directions widely develop:

- maintaining digital archives, collections, libraries (73 structures);

- maintaining digital archives, collections, libraries (73 structures);

- work with data: collecting and digital data research, data visualization and analysis, data modeling, data preservation, database design and implementation, etc. (59 structures);

- digital web publications and electronic editions (34 structures);

- textual research and computerized literary analysis (34 structures);

- human-computer and human-robot interaction, global impact of digital world (33 structures);

- virtual reality, virtual worlds, cyberculture (25);

- creation of online resources, services and platforms (29 structures);

- creation of mobile, multimedia and digital applications (22 structures);

- design and implementation of new digital tools, methods and models (26 structures);

- design and implementation of innovative online tools for education process (21 structures);

- studying of information society, culture and behavior (20 structures);

- digital art, digital documentation, virtual research environments (17 structures by each);

- digital history, social networks, network communications (16 structures by each), 
- digital culture (15);

- artificial intelligence (14);

- digital games (13) and etc.

Covering various subjects, from creation of databases before reconstruction of historical interiors, 3D - modeling of large cultural objects, visualization of existential data, etc., Digital Humanities include:

- use of digital technologies in humanitarian researches, first of all, for processing of big data files: from the analysis of ancient manuscripts ("digital paleography") before studying of literary works and documents of various historical eras ("distant reading" or "digital reading");

- research of features of a new era, sociocultural consequences of digital technologies;

- work with "cultural heritage: "digital art", new media, creation of digital libraries, archives, databases of cultural heritage and museum collections, the digital reconstruction demanding joint efforts of humanists and experts in digital technologies.

The results of research conducted on foreign materials applied to an assessment of the Russian situation. There are a lot more researchers who intentionally ignore IT potential for the humanities and consider that IT destroys modern humanitarian knowledge. Successful researches in the field of branch informatics, based on application IT as tool for researches of traditional material are conducted at many Russian universities and scientific institutes. As working in the direction of the DH centers in Russia the number of universities are defined: Moscow State University, Altai State University, National Research Tomsk State University, Siberian Federal University, Tambov State University, etc.

There were research teams conducting researches in interdisciplinary areas: Humanitarian Informatics, Historical Informatics, Cognitive Sciences, Digital Culture, Social Robotics, etc. Now they feel need for interdisciplinary integration with the research centers in the field of social, computer and natural sciences.

\section{Research development}

Further research will be directed on expansion of a circle of the analyzed DH centers and deepening of the qualitative analysis of the content of their activity that will give the chance:

- to compare the actual problems, investigated within the $\mathrm{DH}$, with the traditional sphere of the humanities;

- to specify classification and to reveal the most perspective directions in development of the DH for medium-term prospect;

- to expand the idea of distribution of the DH centers in the world.

Further research of the methodological bases of development of Digital Humanities will allow to reconstruct the information DH environment, to define the bases for studying of character of information exchanges in which the experts in the field of DH enter.

Creation of the working version of an interactive map "The world centers Digital humanities" on the basis of a prototype will expand the possibilities of scientific communications, will facilitate the creation of conditions for integrative processes in the development of $\mathrm{DH}$, design of interdisciplinary and international research and educational projects. 


\section{Conclusion}

The conducted research allowed to reveal the scale of the DH distribution, to visualize and localize the main structures positioning the belonging to the sphere of $\mathrm{DH}$, to classify the main activities of these structures, to apply a number of methods of computer sciences to carrying out this research on the Russian material.

The study makes it possible to consider the DH as an objective continuation and expansion of the traditional sphere of the humanities, but not a replacement or cancellation of traditional humanitarian requests, to identify promising trends in the development of DH not only for Russia, but also other countries.

\section{Acknowledgements}

The researchers would like to express their gratitude to the Russian Foundation for Humanities for supporting of this research (grant of No. 14-03-00659 "Humanities in the digital age: from branch informatics to Digital Humanities").

\section{References}

1. Humanities in the digital age: from branch informatics to Digital Humanities, Retrieved June 16, 2015, from http://huminf.tsu.ru/our_projects/digital_humanities/ (2014).

2. Journal of Digital Humanities, Retrieved June 16, 2015, from http://journalofdigitalhumanities.org/about/ , (2015).

3. Shurer K., History and Computing or Digital Humanities? Which way to the future? Newsletter of History and Computer Association, 42, 4-8, (2014).

4. Taller M., Discussions around Digital Humanities. Historical informatics. Information technologies and mathematical methods in historical researches and education, 1, 5-13, (2012).

5. Thompson Klein, J., Interdisciplining Digital Humanities: Boundary Work in an Emerging Field. Ann Arbor: University of Michigan Press, (2014).

6. UCLA Digital Humanities, Retrieved June 16, 2015, from http://www.cdh.ucla.edu/about/, (2015).

7. Volodin A.U., The humanities in context of actual digital challenges (review of "digital humanities series" by Michigan university press). Historical informatics. Information technologies and mathematical methods in historical researches and education, 6(4), 99-102, (2013).

8. Volodin A.U., Digital Humanities: interdisciplinarity during a digital era. Newsletter of History and Computer Association, 42, 14-16, (2014). 